\title{
Effort Estimation in Traditional and Agile Mobile Application Development \& Testing
}

\author{
Anureet Kaur ${ }^{1}$, Kulwant Kaur ${ }^{2}$ \\ ${ }^{1}$ I.K.G Punjab Technical University Kapurthala, India \\ ${ }^{2}$ School of IT, Apeejay Institute of Management Technical Campus Jalandhar, India
}

\begin{tabular}{l} 
Article Info \\
\hline Article history: \\
Received Apr 30, 2018 \\
Revised Jul 14, 2018 \\
Accepted Aug 21, 2018 \\
\hline
\end{tabular}

\section{Keywords:}

Agile development

Effort estimation

Mobile software/application

Software engineering

\begin{abstract}
Smartphones/mobile devices are enduring all the aspects of human life. With the significant increase in demand for applications running on smartphones/mobile devices, developers and testers are anticipated to deliver high quality, on time and within budget applications. The estimation of development and testing provides a baseline and act as a tracking gear for stakeholders and developers. There are various approaches for estimation of traditional software development. But mobile applications are considered different from traditional software such as those running on desktop, laptop or on the web. Many traditional estimation techniques used for this software are adapted to mobile domain. With agile software development (ASD) methodology, the scenario of development and estimation has changed drastically and so as mobile app development and estimation. This paper provides a Systematic Literature Review (SLR) on traditional estimation techniques and agile estimation techniques applied in mobile software/application. Also, effort attributes and accuracy parameters for estimation in mobile apps are presented. However, to date, there are very fewer studies done on the mobile application estimation domain using agile methodology.
\end{abstract}

Copyright $\odot 2018$ Institute of Advanced Engineering and Science. All rights reserved.

\section{Corresponding Author:}

Anureet Kaur,

I.K.G Punjab Technical University Kapurthala,India.

Email: anumahal@gmail.com

\section{INTRODUCTION}

Background: - Because of popularity and ease of use of mobile devices has become the most indispensable expedient for human essentials from past few years [1]. Mobile software developers' are driven to release software on time and within budget. For this purpose software estimation plays a very pivotal role in providing the most accurate sizing figure for building confidence in developers and stakeholders relationship [2]. Accuracy in estimation is a crucial factor for planning software development to evade budget overruns and tardy delivery of software. Estimation of test effort along with development is considered crucial for apprehending quality software [3]. Development of mobile software is considered different from developing traditional software pertaining to its distinctive features such as limited memory, processing power, small and multiple input interface, multiple connections, bandwidth factor, a lower battery, etc. [4-7].

With the advent of Agile Software Development (ASD) [8-9], entire software development community has been driven to the adoption of agile methodology. The Agile espousal to mobile application development is considered as a natural fit by many researchers [10-14]. The effort estimation of software development in an agile environment is also different from traditional software estimation [8]. Effort estimation is more exigent in an agile because of employing dynamic changes in requirements [15]. There are many estimation techniques for traditional software estimation in an agile environment such as planning poker, Expert judgment, Use Case Points (UCP) Method, etc. [8, 16]. 
Mobile applications are different from traditional software [4-7] and agile approach to mobile application development and estimation needs a solid methodological approach for predicting effort.

The Problem: - The approaches used for estimation of traditional software are adapted for mobile application development. But presently, not much work has been dedicated to identifying suitable approaches exclusively for effort estimation of mobile apps [17]. The estimation techniques in agile software development for traditional software cannot be adapted to mobile domain pertaining to different characteristics of mobile apps. However, there is very less literature available on the estimation of mobile apps in an agile environment

Proposed solution: - This paper contributes mainly by examining the state-of-art of techniques applied for estimation of mobile software/applications in traditional software development and agile software development by using Systematic Literature Review (SLR). SLR will form baselines for mobile app developers for the selection of appropriate estimation method according to their need. This will also help researchers in filling the gap by proposing formal estimation models for mobile apps considering its specific characteristics.

The rest of this paper is ordered as follows: Section 2 presents the Research method used for conducting the Systematic Literature Review (SLR). Section 3 presents the results of SLR and some discussions on selected studies and finally, Section 4 concludes the paper with future directions.

\section{RESEARCH METHOD}

The goal of this study is to gain an understanding of the current state-of-art in mobile app development effort estimation. To this end, an explorative study is conducted using the Systematic Literature Review (SLR) succeeding the guidelines postulated by Kitchenhan and Charters [18]. SLR is a research method for carrying out a literature review in a systematic way of charting well-defined phases. SLR method uses three phases for performing literature review including Planning and specifying research questions as a first phase, the second phase is conducting the review that comprises an identification of search string \& data sources, selecting studies \& data extraction and the third one being results reporting.

\subsection{Planning Phase}

For the smooth conduct of systematic literature review, proper planning is necessary. The research questions derive from the entire systematic literature review planning phase.

\subsubsection{Research Questions (Rqs)}

Affirming the research questions is the vital part of any systematic review. In accordance with guidelines proposed by Petticrew and Roberts [19], the criteria to frame research questions are based on PICOC (Population, Intervention, Comparison, Outcomes, and Context). If the research question is not outlined properly, the literature review may turn out off the course. For this study PICOC are defined as:-

Population: Mobile Application projects

Intervention: Effort estimation techniques/methods/process

Comparison: Traditional mobile app effort estimation techniques with an agile mobile app estimation.

Outcomes: Estimation models to follow for mobile app development in agile and traditional software development.

Context: Review the existing studies on estimation of mobile apps.

The research questions steer the entire systematic review methodology. The major objective of this finding is to answer the following research question:-

RQ1. What are the traditional effort estimation techniques used for mobile software/application development and testing?

RQ2. What is currently known effort estimation techniques followed in agile mobile software/application development and testing?

RQ3. What are the estimation attributes and accuracy parameters used in estimation techniques for mobile application?

\subsection{Conducting the Review Phase}

\subsubsection{Search Strategy}

The aim of directing search strategy is to identify studies pertaining to answer the RQs. Further, search strategy can be conducted in three phases; Identifying keywords and Defining Search strings, Identifying the data sources and Search Process in Data sources. 


\section{a) Identifying keywords and Defining Search Strings}

The first phase comprises forming the search string. The search strategy is set up to describe search strings and primary data sources. The guidelines provided by [18] are followed to define the search string by analyzing the main keywords in RQs, synonyms of the keywords and on any other spellings of the words. The identified keywords are shown in Table 1.

Table 1. Identified Keywords and synonymous

\begin{tabular}{cc}
\hline Keywords & Synonymous Terms \\
\hline Software & Software, project, system, application \\
Effort & Cost, resource, size, metric \\
Estimation & Estimating, estimate, prediction, predicting, predict, assessment, forecasting, forecast, calculation, \\
& calculate, calculating, sizing, measure, measuring \\
Mobile Application & Mobile software, Mobile Apps, Mobile project \\
Development & Improvement, Progress \\
Testing & Test, verification, validation \\
Agile & Scrum, XP, lean, crystal \\
Method & Process, techniques, models, approaches \\
\hline
\end{tabular}

Based on the identified keywords, the search string is obtained by joining synonymous terms using the logical 'OR', other keywords using logical 'AND' and wildcard character ('*'). Here wildcard character represents 0,1 , or any number of alphanumeric characters. The following search string is obtained:

("Mobile Application" OR "Mobile software" OR "Mobile App" OR "Mobile project") AND ("Develop*") AND ("estimate*" OR "predict*" OR "assessment" OR "forecast*" OR "calculate*" OR "sizing" OR "measure*") AND ("Improvement" OR "Progress" AND "Process" OR "techniques" OR "models" OR "approaches") AND ( "agile" OR "scrum" OR "XP" OR "lean" Or "crystal")

\section{b) Data sources}

The digital databases that are used to search the keywords are SpringerLink, IEEE Xplore, ACM Digital Library, Elsevier Science Direct, Wiley InterScience and Google Scholar.

\section{c) Search Process in Data sources}

The next phase is to apply the search string to chosen electronic data sources to find all the entailed studies. This phase is divided into two sub-phases: primary and secondary search phase. In Primary Search Phase, the electronic data sources identified are searched based on the search string defined earlier. The results from data sources are monitored to include search string in title and abstracts. The search string is again refined each time to check the outcome and analyzed for better results. The aim of this maiden check is to evaluate the efficacy of the search string. Additionally, results are restricted to peer-reviewed conference papers and journal articles which are available between 2008 and 2018 (last decade). The duplicate titles and abstracts are removed. In the secondary search phase, a technique called snowball tracking is used for studying all the references of primary studies to exploit further studies and increase the chances of inclusion of important papers in the systematic literature review. Table 2 lists the refined results from data sources after primary and secondary search phase.

Table 2.Overview of search results

\begin{tabular}{cc}
\hline Data Sources & Relevant Search Results \\
\hline SpringerLink & 53 \\
IEEE Xplore & 75 \\
ACM Digital Library & 57 \\
Elsevier Science Direct & 66 \\
Google Scholar & 83 \\
Wiley InterScience & 20 \\
Total & 354 \\
\hline
\end{tabular}

\subsubsection{Inclusion/Exclusion Criteria for Selecting Studies}

The results acquired through search string defined previously in the electronic databases are analyzed according to the Inclusion/Exclusion criteria. The evaluation of the papers is done by reading the title and abstract first and checked if it is related to the issues addressed in RQs. Then the decision is made for its acceptance for reading the whole paper or is rejected therein. The inclusion criteria: 
1. Studies that include estimation methods of mobile application development AND;

2. Described in English AND;

3. Peer-reviewed papers are selected.

The exclusion criteria:

1. Studies having mobile application development process and not are not considering estimation OR;

2. Not described in English OR;

3. Not peer reviewed

\subsubsection{Quality Assessment and Data Extraction}

To evaluate the quality of shortlisted studies; 6 questions are prepared to be answered for each selected study. The question can be answered as ' $\mathrm{Y}$ ', ' $\mathrm{M}$ ' or ' $\mathrm{N}$ '. The scores for ' $\mathrm{Y}$ ' $=1$, ' $\mathrm{P}$ ' = 0.5 , and ' $\mathrm{N}$ ' = 0 . The questionnaire is developed by using the guidelines defined by Kitcheham and Charters [18]. The quality score for each paper should be minimum 3 to be further included in the study.

Following are the questions in the questionnaire:

1. Are the research motives clearly stated?

2. Was the study designed to achieve the aims?

3. Are the estimation techniques well defined?

4. Is the research process documented adequately?

5. Are all research questions answered adequately?

6. Are the main findings stated clearly in terms of creditability, validity, and reliability?

The authors carried out a quality assessment for the all selected studies. Four studies are excluded owned by their low-quality score. The data extraction phase involves extraction of data of the final selected studies that address the peculiarities of RQ. The data extraction for all conclusive selected studies is done in an MS Excel sheet containing: - Paper name, year of publication, author' name, and paper URL.

\section{RESULTS AND DISCUSSION}

The results of the Systematic Literature Review (SLR) and answers for Research Questions (RQs) are presented in this section. Table 3 describes the studies that are selected after full screening and passing quality assessment criteria.

Table3. Selected papers for final studies

\begin{tabular}{ccccccccc}
\hline Study ID & Reference ID & Year & Study ID & Reference ID & Year & Study ID & Reference ID & Year \\
\hline S1 & {$[20]$} & 2013 & S2 & {$[21]$} & 2015 & S3 & {$[22]$} & 2015 \\
S4 & {$[23]$} & 2014 & S5 & {$[24]$} & 2016 & S6 & {$[25]$} & 2013 \\
S7 & {$[26]$} & 2014 & S8 & {$[27]$} & 2016 & S9 & {$[28]$} & 2017 \\
S10 & {$[29]$} & 2017 & S11 & {$[30]$} & 2014 & S12 & {$[31]$} & 2016 \\
S13 & {$[32]$} & 2014 & S14 & {$[33]$} & 2008 & S15 & {$[34]$} & 2014 \\
S16 & {$[35]$} & 2013 & S17 & {$[36]$} & 2015 & S18 & {$[37]$} & 2017 \\
S19 & {$[38]$} & 2017 & S20 & {$[39]$} & 2018 & S21 & {$[40]$} & 2016 \\
\hline
\end{tabular}

\subsection{Traditional Techniques for Estimating Mobile Application Development: RQ1}

Sixteen studies out of twenty-one selected studies investigated the traditional estimation techniques for mobile applications. Table 4 lists the identified techniques where an agile approach is not followed for development of mobile apps. The techniques are broadly classified into three categories [20] i.e. Algorithmic-based models, Expert Judgment based models and analogy based models. COSMIC Function Size Measurement [21-28] is frequently used for estimation technique which is used to measure the functional size of the mobile app. Other types of estimation techniques identified are Function Point Analysis [29, 30] and Use Case Point [31] which is algorithmic-based models that measure functional, technical factors and environmental factors for estimation. Regression-Based technique [32] uses a parametric model based on effort predictors and data points collected through an online questionnaire which are further used in the regression model. Delphi method [33] is based on experience to estimate the effort whereas Architecture Based estimation model [34] for reliability and testing estimation of the mobile application is proposed and the case study was conducted in two companies. Another algorithmic approach for estimating the cost of developing Android mobile apps are based on the COCOMO -I and II model [35]. Analogy-based estimation plus functional size measurement [36] approach is also proposed for mobile apps. 
Table 4. Traditional Estimation Techniques for mobile applications

\begin{tabular}{ccc}
\hline Estimation Techniques & Study ID & Approach Type \\
\hline COSMIC Function Size Measurement & S1,S2,S3,S4,S5,S7,S11,S21 & Algorithmic-based Model \\
Function Point Analysis & S6, S15 & Algorithmic-based Model \\
Delphi method & S9 & Expert Judgment \\
Use Case Point & S10 & Algorithmic-based Model \\
Mybrid (Analogy based estimation + Function Size & S13 & Analogy and Algorithmic based \\
Measurement) & model \\
Regression-Based & S12 & Algorithmic-based Model \\
Architecture-Based & S14 & Algorithmic-based Model \\
COCOMO -I and II & S8 & Algorithmic-based Model \\
\hline
\end{tabular}

\subsection{Agile techniques for estimating mobile application development: RQ2}

Agile approach to mobile application development estimation has very less number of studies. One of the reason could be the adoption of agile to mobile context is still in its evolving phase and many practitioners are adapting traditional estimation methodologies to an agile environment for mobile apps. The identified studies are listed in table 5. Traditional use case point method of estimation is extended by adding efficiency and risk factor of testers in the agile team [37]. Another technique [38] is based on a stepwise linear regression model which estimates the effort for Android apps from requirements specification including a number of use cases, actors, etc. User story point [39] is refined by considering additional factors along with size and complexity. The quality factor, Novelty factor and Type factor of User Story are added to deliver the best estimation for mobile app development. Another approach [40] uses Early Use Case Point (EUCP) and Extended Use Case Point (EXUCP) along with COCOMO drivers at different iteration levels in agile mobile app development. An experience-driven approach using the Delphi technique [41] is used for effort estimation having a mobile app as one of the case studies.

Table 5. Agile Estimation Techniques for mobile applications

\begin{tabular}{ccc}
\hline Agile Estimation Techniques & Study ID & Approach Type \\
\hline Use Case Point & S16 & Algorithmic-based models \\
Step-wise Linear Regression & S17 & Algorithmic-based models \\
User story Point & S18 & Expert Judgment \\
Use Case Point+ COCOMO & S19 & Algorithmic-based models \\
Delphi & S20 & Expert Judgment \\
\hline
\end{tabular}

\subsection{Estimation Attributes and Accuracy Parameters for Mobile Apps: RQ3}

The estimation attributes identified in the selected studies are mostly focused on size metric whether based on use case, function point and story point. Table 6 lists the other estimation attributes that are identified for estimation. Table 7 lists the parameters used to assess the accuracy of estimation of mobile applications. MMRE and Pred(x) are highly followed in most of the studies.

Table 6. Estimation attributes for mobile applications

\begin{tabular}{|c|c|}
\hline Estimation Attributes & Study ID \\
\hline Size & S1,S2,S3,S4,S5.S6,S7,S10,S11,S13,S15,S16,S17,S18(user stories),S19,S21 \\
\hline Cost & $\mathrm{S} 8, \mathrm{~S} 13, \mathrm{~S} 19$ \\
\hline Others & $\begin{array}{c}\text { S9(Score Metric), S12(Mean and SD of collected mobile apps variables), S14(architecture } \\
\text { based), S20(mean effort based on experience) }\end{array}$ \\
\hline
\end{tabular}

Table 7. Parameters for measuring the accuracy of estimation

\begin{tabular}{cc}
\hline Accuracy Parameters & Study ID \\
\hline MRE(Magnitude of Relative Error) & S2,S3,S18 \\
MMRE(Mean Magnitude of Relative Error) & S2,S3,S12,S18,S19 \\
MdMRE(Median MRE) & S2,S3, S12 \\
Pred(percentage relative error deviation) & S2,S3, S12,S18,S19 \\
Linear Regression (R2) & S12,S19 \\
Not Defined & S1,S4,S6,S7,S9S,10,S13,S14,S20,S21 \\
Others & S8(web-based survey), S11(Compared with actual effort), S15(Compared \\
& with actual effort), S16(Comparison with actual effort), S17(compared with \\
source code as a software measure)
\end{tabular}




\section{CONCLUSION}

This paper represents a Systematic Literature Review (SLR) concerning effort estimation techniques in mobile software/applications that are developed either using traditional software development or agile software development methodology. Initially, the search string produced a total of 354 results and only 23 papers are selected for primary studies. Two more papers are included after performing secondary search process but four papers are excluded due to low-quality scores in quality assessment criteria. The findings of this SLR are founded on 21 papers whose data are extracted into an excel sheet and then excavated to answer the RQs formed during the planning phase.

The traditional estimation techniques applied in mobile applications are COSMIC Function Size Measurement, Function Point Analysis, Delphi method, Use Case Point, Hybrid(Analogy based estimation plus Function Size Measurement), Regression-Based, Architecture Based and COCOMO -I and II.

The agile estimation techniques in the mobile domain are very few. The identified techniques are adaption or modification of traditional estimation techniques used in the agile environment to mobile software. The techniques are Use Case Point, Step-wise Linear Regression, User story Point with additional factors, Use Case Point plus COCOMO and Delphi technique. SLR also presented effort attributes and accuracy measures from each selected study used for assessing estimation accuracy.

From the current studies, it can be concluded that the adoption of agile software development in mobile domain is proliferating from last decade [42-43] and hence there is a dire need for formal estimation models for mobile software. The mobile software characteristics played a critical role during estimation in traditional software development [4-7], [44]. It is presented in SLR that none of the estimation models for agile mobile application development are cogitating specific characteristics of mobile apps. This research gap provides ideas for researchers to devise novel models for estimation of mobile apps in agile context or extend existing estimation approaches of estimation.

\section{ACKNOWLEDGMENT}

The authors are thankful to the Department of RIC, I.K.G. Punjab Technical University, Kapurthala, Punjab, India and providing an opportunity to carry out this research work.

\section{REFERENCES}

[1] I. Malavolta, S. Ruberto, T. Soru, and V. Terragni. "End users' perception of hybrid mobile apps in the Google Play Store". IEEE Int. Conf. Mobile Services, New York, NY, USA, Jun./Jul. 2015, pp. 25-32.

[2] Y. M. G. Soares and R. A. d. A. Fagundes. "Software time estimation using regression methods". IEEE Latin American Conference on Computational Intelligence (LA-CCI), Arequipa, 2017, pp. 1-6.

[3] Pulak Sahoo, J. R. Mohanty, "Early Test Effort Prediction using UML Diagrams”, Indonesian Journal of Electrical Engineering and Computer Science(IJEECS). 2017; 5(1): pp. 220 - 228

[4] L. Corral, A. Sillitti and G. Succi. "Software development processes for mobile systems: Is agile really taking over the business?", 1st International Workshop on the Engineering of Mobile-Enabled Systems (MOBS), San Francisco, CA, 2013, pp. 19-24.

[5] de Souza L.S., de Aquino G.S. "Mobile Application Estimate the Design Phase". In: Maciaszek L., Filipe J. (eds) Evaluation of Novel Approaches to Software Engineering. Communications in Computer and Information Science, vol 551. Springer, Cham

[6] Inukollu VN, Keshamoni DD, Kang T, Inukollu M. "Factors influencing quality of mobile apps: Role of mobile app development lifecycle". arXiv preprint arXiv:1410.4537. 2014 Oct 16.

[7] Flora HK, Wang X, Chande SV. "An investigation on the characteristics of mobile applications: A survey study". International Journal of Modern Education and Computer Science. 2014 Oct; 6(6).

[8] Muhammad Usman, Emilia Mendes, Francila Weidt, and Ricardo Britto. 2014. "Effort estimation in agile software development: a systematic literature review". In Proceedings of the 10th International Conference on Predictive Models in Software Engineering (PROMISE 2014). ACM, New York, NY, USA, 82-91.

[9] Rajesh H Kulkarni, P Padmanabham, Manasi Harshe, K K Baseer, Pallavi Patil, "Investigating Agile Adaptation for Project Development", International Journal of Electrical and Computer Engineering (IJECE), 2017; 7 (3): 1278 1285.

[10] Abrahamsson, P., Hanhineva, A., Hulkko, H., Ihme, T.,Jäälinoja, J., Korkala, M., Koskela, J., Kyllönen, P., Salo, O. "Mobile-D: An Agile approach for mobile application development". In proceedings of OOPSLA'04. 2004.

[11] Jeong, Y.J., Lee, J.H., Shin, G.S. "Development process of mobile application SW based on Agile methodology". 10th Intl. Conf. on Advanced Communication Tech., pp. 362-366. 2008.

[12] Rahimian, V. Ramsin, R. "Designing an Agile methodology for mobile software development: A hybrid method engineering approach". 2nd International Conference on Research Challenges in Information Science, 2008, pp. 337-342.

[13] Scharff, C., Verma, R. "Scrum to support mobile application development projects in a just-in-time learning context". In Proc. of the ICSE Workshop CHASE 2010, pp. 25-31. 
[14] Cunha, T., Dantas, V., Andrade, R. "SLeSS: A Scrum and Lean Six Sigma integration approach for the development of software customization for mobile phones". 25th Brazilian Symposium on Software Engineering, pp. 283-292. 2011.

[15] Binish Tanveer, Liliana Guzmán, and Ulf Martin Engel. "Understanding and improving effort estimation in Agile software development: an industrial case study". In Proceedings of the International Conference on Software and Systems Process (ICSSP '16). ACM, New York, NY, USA, 41-50.

[16] B. Prakash, V. Viswanathan.A, "Survey on Software Estimation Techniques in Traditional and Agile Development Models". Indonesian Journal of Electrical Engineering and Computer Science(IJEECS) .2017; 7(3): 867 -876.

[17] A. I. Wasserman. "Software engineering issues for mobile application development". The FSE/SDP workshop on Future of software engineering research. ACM, 2010, pp. 397-400

[18] B. Kitchenham, S. Charters. "Guidelines for Performing Systematic Literature Reviews in Software Engineering". EBSE Technical Report, EBSE-2007-2101, 2007

[19] Petticrew, M. \& Roberts, H. "Systematic Reviews in the Social Sciences: A Practical Guide", Blackwell Publishing. 2006.

[20] E. Mendes. "Cost Estimation Techniques for Web Projects". IGI Global. 2007.

[21] A. Nitze. "Measuring mobile application size using cosmic FP". In DASMA Metrik Kongress, vol. 11, 2013.

[22] L. D'Avanzo, F. Ferrucci, C. Gravino, and P. Salza. "Cosmic functional measurement of mobile applications and code size estimation”. In Proceedings of the 30th Annual ACM Symposium on Applied Computing. ACM, 2015, pp. $1631-1636$

[23] F. Ferrucci, C. Gravino, P. Salza, and F. Sarro. "Investigating functional and code size measures for mobile applications: A replicated study". In International Conference on Product-Focused Software Process Improvement. Springer, 2015, pp. 271-287.

[24] N. A. S. Abdullah, N. I. A. Rusli, and M. F. Ibrahim. "Mobile game size estimation: Cosmic fsm rules, uml mapping model and unity3d game engine”. In Open Systems (ICOS), 2014 IEEE Conference on. IEEE, 2014, pp. 42-47.

[25] A. Sellami, M. Haoues, H. Ben-Abdallah, A. Abran, A. Lesterhuis, C. Symons, and S. Trudel. "Sizing natural language/uml requirements for web and mobile applications using cosmic fsm". Tech. Rep., 2016.

[26] H. van Heeringen and E. Van Gorp. "Measure the functional size of a mobile app: Using the cosmic functional size measurement method". In Software Measurement and the International Conference on Software Process and Product Measurement (IWSM-MENSURA), 2014 Joint Conference of the International Workshop on. IEEE, 2014, pp. 11-16.

[27] Laudson Silva de Souza and Gibeon Soares de Aquino Jr. "MEFFORTMOB: A Effort Size Measurement for Mobile Application Development”. International Journal of Software Engineering \& Applications (IJSEA) .2014: 63-81.

[28] Vogelezang, Frank, Jayakumar Kamala Ramasubramani and Srikanth Arvamudhan. "Estimation for Mobile and Cloud Environments". Modern Software Engineering Methodologies for Mobile and Cloud Environments. IGI Global, 2016. 61-87.

[29] T. Preuss. "Mobile applications, functional analysis, and the customer experience". The IFPUG Guide to IT and Software Measurement, IFPUG, Ed. Boca Raton FL, USA: Auerbach Publications, 2012, pp. 408-433.

[30] Tunali V. "Software Size Estimation Using Function Point Analysis-A Case Study for a Mobile Application". Muhendisik ve Teknoloji Sempozyumu. 2014.

[31] Mariem Haoues, Asma Sellami, and Hanene Ben-Abdallah. "A rapid measurement procedure for sizing web and mobile applications based on COSMIC FSM method". In: Proceedings of the 27th International Workshop on Software Measurement and 12th International Conference on Software Process and Product Measurement (IWSM Mensura '17). ACM, New York, NY, USA, 129-137.

[32] S. A. Shahwaiz, A. A. Malik and N. Sabahat. "A parametric effort estimation model for mobile apps". In 19th International Multi-Topic Conference (INMIC), Islamabad, 2016, pp. 1-6.

[33] Catolino, G., P. Salza, Gravino, C., \& Ferrucci, F. "A set of metrics for the effort estimation of Mobile apps". Proceedings of the IEEE/ACM 4th Int. Conf. Mob. Softw. Eng. Syst. MOBILESoft.2017.

[34] Wadhwani V, Memon F, Hameed MM. "Architecture based reliability and testing estimation for mobile applications". In International Multi Topic Conference 2008 Apr 11 (pp. 64-75). Springer, Berlin, Heidelberg.

[35] Zubair Asghar M, Habib A, Habib A, Rabail Zahra S, Ismail S. "AndorEstimator: Android-based Software Cost Estimation Application”. arXiv preprint arXiv:1605.02304. 2016.

[36] A. Nitze, A. Schmietendorf, R. Dumke. "An Analogy-Based Effort Estimation Approach for Mobile Application Development Projects". In Software Measurement and the International Conference on Software Process and Product Measurement (IWSM-MENSURA), 2014.

[37] Parvez, A.W.M.M. "Efficiency factor and risk factor based user case point test effort estimation model compatible with agile software development". In Proceedings of the International Conference on Information Technology and Electrical Engineering - ICITEE'13. Yogyakarta, Indonesia .2013. pp. 113-118.

[38] R. Francese, C. Gravino, M. Risi, G. Scanniello, G. Tortora. "On the use of requirements measures to predict software project and product measures in the context of Android mobile apps: A preliminary study". In Proc. 41st Euromicro Conf. Series Softw. Eng. Advanced Appl. 2015. pp. 357-364.

[39] W. Aslam, F. Ijaz, M. I. Lali, and W. Mehmood, "Risk-aware and quality enriched effort estimation for mobile applications in distributed agile software development". J. Inf. Sci. Eng. 2017; 33(6): 1481-1500

[40] K. Qi and B. W. Boehm. "A light-weight incremental effort estimation model for use case driven projects". IEEE 28th Annual Software Technology Conference (STC), Gaithersburg, MD, 2017, pp. 1-8. 
[41] Lusky M., Powilat C., Böhm S. "Software Cost Estimation for User-Centered Mobile App Development in Large Enterprises". In: Ahram T., Karwowski W. (eds) Advances in Human Factors, Software, and Systems Engineering. AHFE 2017. Advances in Intelligent Systems and Computing, vol 598. Springer, Cham

[42] Corral, L., Sillitti, A., \& Succi, G. "Agile software development processes for mobile systems: Accomplishment, evidence and evolution”. In Proceedings of the Mobile Web Information Systems. 2013. 90-106.

[43] Rita Francese, Carmine Gravino, Michele Risi, Giuseppe Scanniello, and Genoveffa Tortora. "Mobile app development and management: results from a qualitative investigation". In Proceedings of the 4th International Conference on Mobile Software Engineering and Systems. 2017. IEEE Press, Piscataway, NJ, USA, 133-143.

[44] Kumar, N. A., Krishna, K. T. H., \& Manjula, R. "Challenges and best practices in mobile application development". Imp. J. Interdiscip. Res.2016; 2(12): 1607-1611. 